\title{
INFLUÊNCIA DA VARIAÇÃO DA PRODUTIVIDADE DAS USINAS HIDRELÉTRICAS NO CÁLCULO DA ENERGIA FIRME
}

\author{
Edimar J. de Oliveira* \\ edimar.oliveira@ufjf.edu.br \\ Rafael Santos Rocha* \\ rafaeledourado.net \\ Ivo Chaves da Silva Jr.* \\ ivo.junior@ufje.edu.br
}

\author{
André Luis Marques Marcato* \\ andre.marcato@ufjf.edu.br
}

Leonardo Willer de Oliveira*

leonardowillereyahoo.com

José L. R. Pereira*

jluiz@ieee.org

\author{
*Universidade Federal de Juiz de Fora \\ Campus UFJF - Faculdade de Engenharia - Juiz de Fora, MG, Brasil
}

\begin{abstract}
In the present work, the problem associated to the firm energy evaluation is treated as a non linear optimization model, which allows the representation of the productivity variation of the hydro plants. The proposed model takes into account the individualized representation of the plants and the historical series of flows since the month of January of 1931. The proposed optimization problem will be solved using the Primal-Dual Interior Point Method. A case study will be presented including the Brazilian Interconnected National System. The results obtained show that the proposed methodology is promising, since it presents an energy market value more realistic when compared with existing methodologies.
\end{abstract}

KEYWORDS: Firm Energy, Efficient Storage, Nonlinear Optimization, Interior Point Method.

\section{RESUMO}

No presente trabalho, o problema associado ao cálculo da energia firme é tratado como um modelo não linear de otimização, o que permite a representação da variação da produtividade das usinas. No modelo proposto é considerada a representação individualizada das usinas bem como a série

Artigo submetido em 22/02/2008 (Id.: 00857)

Revisado em 17/06/2008, 13/11/2008, 18/12/2008

Aceito sob recomendação do Editor Associado Prof. Eduardo N. Asada histórica de vazões desde o mês de janeiro de 1931. O problema de otimização proposto é resolvido através do método primal-dual de pontos interiores. Adicionalmente, é apresentado um estudo de caso abrangendo o Sistema Interligado Nacional Brasileiro. Os resultados obtidos mostram que a metodologia proposta é promissora, tendo em vista que apresenta um valor de mercado de energia mais realista quando comparado com outras metodologias.

PALAVRAS-CHAVE: Energia Firme, Armazenamento Eficiente, Otimização Não Linear, Método de Pontos Interiores.

\section{INTRODUÇÃO}

Um dos desafios da área de planejamento da operação e expansão consiste em determinar a melhor metodologia para avaliar a capacidade de suprimento de um sistema hidrelétrico e das usinas que o compõem frente um comportamento hidrológico estocástico, denominado energia firme. Tradicionalmente, avalia-se tanto a energia firme global do sistema (conjunto de usinas hidrelétricas), como também, a energia firme individualizada para cada usina hidrelétrica que compõe o sistema.

De acordo com as regras de comercialização de energia elétrica no Brasil, cada usina hidrelétrica tem um limite (lastro) para o estabelecimento de contratos. Obviamente, o lastro não corresponde à capacidade instalada da usina hidrelétrica, 
visto que a geração é fortemente influenciada pela hidrologia. O lastro ou garantia física ou energia assegurada é calculado admitindo-se um risco de 5\% (Soares \& Marcato, 2006), (Hreinsson \& Barroso, 2004).

A metodologia para o cálculo da energia assegurada no Brasil é baseada em sistemas equivalentes de energia para cenários sintéticos de energias afluentes (Arvanitids \& Rosing, 1970). A energia assegurada é calculada por um modelo computacional baseado em programação dinâmica dual estocástica (PDDE) (Pereira et al., 1998).

Logo, a energia assegurada é inicialmente determinada para um sistema equivalente de energia (ou conjunto de usinas). A energia firme individualizada das usinas hidrelétricas é utilizada como fator de rateio da energia assegurada global de um sistema para as usinas hidrelétricas que o compõem (Camargo \& Mendes, 2003), (Mendes et al., 2003). Portanto, a energia firme individual influencia diretamente a determinação da garantia física das usinas (ou lastro) a fim de estabelecer seus contratos com as distribuidoras, consumidores livres e comercializadores (Mendes \& Santana, 2003), (Faria et al., 2006). Por este motivo, torna-se extremamente importante a avaliação de novas metodologias para o cálculo da energia firme.

A energia firme do sistema é definida como a máxima capacidade de suprimento de energia de um conjunto de usinas hidrelétricas sem a ocorrência de déficits, considerando-se todo o registro histórico de afluências (Hicks et al., 1974). Por outro lado, imaginando-se um mercado igual à energia firme do sistema, a energia firme de cada usina é determinada pela geração média da usina durante o período crítico. O período crítico é dado pelo período compreendido entre um mês onde se observa a máxima energia armazenada no sistema e o mês onde se observa a menor energia armazenada no sistema sem re-enchimentos intermediários.

O cálculo da energia firme é um problema complexo devido à interconexão de usinas em cascata em uma mesma bacia hidrográfica e principalmente, devido as não linearidades inerentes ao problema de despacho hidráulico (Silva Fo. et al., 2003), (Yu et al., 1998), (Yeh, 1985). As não linearidades intrínsecas ao problema estão associadas às perdas hidráulicas, polinômios cota-volume, cota-área e vazão-nível jusante (Marques et al., 2006), (Soares \& Carneiro, 1991).

A referência (Soares \& Marcato, 2006) propõe uma metodologia de cálculo da energia firme baseada em programação linear, onde a solução é obtida através de um único problema de programação linear, no qual todos os estágios mensais e todas as usinas são representadas. Adicionalmente, para modelar a varaiação da produtividade das usinas, foi desenvolvido um programa baseado em programação linear seqüencial (PLS). Este modelo faz o cálculo das produtividades das usinas baseado nos resultados obtidos na iteração anterior. No entanto, foi observado um grande esforço computacional, pois o método realiza sucessivas execuções do problema de programação linear.

A referência (Cramton \& Stoft, 2007) mostra que o conceito de energia firme também é utilizado no mercado de energia da Colômbia influenciando fortemente as relações contratuais entre os agentes, sinalizando adequadamente a remuneração dos mesmos de forma a atrair investimentos ao setor, o que confere maior confiabilidade e eficiência ao modelo operacional do mercado de energia.

Atualmente, o cálculo da energia firme para as usinas do Sistema Brasileiro é realizado por um modelo desenvolvido pelo Centro de Pesquisas de Energia Elétrica (CEPEL). Este modelo, denominado SUISHI-O, faz uso da representação individualizada das usinas hidrelétricas e de heurísticas operativas. Embora os resultados obtidos mostrem uma elevada robustez metodológica e um reduzido esforço computacional, a energia firme alocada entre as usinas pode não retratar o ponto de ótimo de operação global (Soares \& Marcato, 2006).

No presente trabalho, o problema associado ao cálculo da energia firme é tratado através de um modelo não linear de otimização, o que permite a representação não linear da variação da produtividade das usinas. A representação individualizada das usinas é utilizada juntamente com a série histórica de vazões desde o mês de janeiro de 1931. O problema de otimização não linear proposto é resolvido através do método primal-dual de pontos interiores (Granville, 1994), (Karmarkar, 1984). Para testar a metodologia não linear proposta, será utilizado um estudo de caso abrangendo o Sistema Interligado Nacional (SIN), contendo 107 usinas hidrelétricas. Os resultados obtidos serão apresentados e discutidos a fim de mostrar a eficácia da metodologia proposta.

\section{FORMULAÇÃO PROPOSTA}

O problema é formulado como:

$$
\max \mathrm{EF}
$$

Sujeito a:

$$
\begin{gathered}
V_{i}^{t}+Q_{i}^{t}+S_{i}^{t}-V_{i}^{t-1}-\sum_{m \in M_{i}}\left(Q_{m}^{t}+S_{m}^{t}\right)-A_{i}^{t}=0\left(\lambda_{h_{i}^{t}}^{t}\right) \\
\sum_{i=1}^{N H} P G_{i}^{t}-E F=0\left(\lambda_{d}{ }^{t}\right)
\end{gathered}
$$




$$
\begin{gathered}
S_{i \min }^{t} \leq S_{i}^{t} \leq \infty \\
Q_{i \min }^{t} \leq Q_{i}^{t} \leq Q_{i \max }^{t} \\
V_{i \min }^{t} \leq V_{i}^{t} \leq V_{i \max }^{t}
\end{gathered}
$$

onde,

EF Representa a energia firme total do sistema [MW];

$V_{i}^{t}$ Representa o volume armazenado no reservatório da usina $i$, durante o estágio $t\left[\mathrm{hm}^{3}\right]$;

$Q_{i}^{t}$ Representa o volume turbinado na usina $i$, durante o estágio $t\left[\mathrm{hm}^{3} / \mathrm{mês}\right]$;

$S_{i}^{t}$ Representa o volume vertido na usina $i$, durante o estágio $t\left[\mathrm{hm}^{3} / \mathrm{mês}\right]$;

$M_{i}$ Representa o conjunto de usinas imediatamente a montante da usina $i$

$A_{i}^{t}$ Representa a afluência incremental da usina $i$, durante o estágio $t\left[\mathrm{hm}^{3} / \mathrm{mês}\right]$;

$\lambda_{h i}^{t}$ Representa o multiplicador de Lagrange associado a cada equação de balanço hídrico no modelo de otimização;

NH Representa o total de usinas hidrelétricas consideradas no estudo;

$P G_{i}^{t}$ Representa a geração de energia na usina $i$, durante o estágio $t[\mathrm{MW}]$;

$\lambda_{d}{ }^{t}$ Representa o multiplicador de Lagrange associado a cada equação de balanço de demanda no modelo de otimização;

$S_{i_{\text {min }}}^{t}$ Representa o volume vertido mínimo no vertedouro da usina $i$, durante o estágio $t\left[\mathrm{hm}^{3} / \mathrm{mês}\right]$;

$Q_{i_{\text {min }}}^{t}$ Representa o volume turbinado mínimo da usina $i$, durante o estágio $t\left[\mathrm{hm}^{3}\right]$;

$V_{i \text { min }}^{t}$ Representa o volume armazenado mínimo no reservatório da usina $i$, durante o estágio $t\left[\mathrm{hm}^{3} / \mathrm{mês}\right]$;

$Q_{i_{\max }}^{t}$ Representa o volume turbinado máximo da usina $i$, durante o estágio $t\left[\mathrm{hm}^{3}\right]$;

$V_{i}^{t}{ }_{\max }$ Representa o volume armazenado máximo no reservatório da usina $i$, durante o estágio $t\left[\mathrm{hm}^{3} / \mathrm{mês}\right]$.

A função objetivo (1) corresponde à máxima demanda a ser atendida, ou seja, o máximo mercado de energia que pode ser atendido com $0 \%$ de risco.
A equação (1.1) representa a conservação da água nos reservatórios, também chamada de restrição de balanço hidráulico.

A equação (1.2) representa a conservação de energia associada a capacidade das usinas de atendimento à demanda.

As restrições (1.3)-(1.5) definem os limites operativos inferior e superior das usinas hidrelétricas. Estas restrições são tratadas através do método primal-dual de pontos interiores proposto por (Granville, 1994). Neste modelo de tratamento dos limites de canalização, as variáveis de folga correspondentes aos limites inferiores (sl) e aos limites superiores (su), introduzidas pelo método de pontos interiores, através da função barreira logaritmica, são tratadas fora da matriz Henssiana $(\mathrm{W})$.

A função lagrangeana (L) correspondente a este problema é apresentada em (2), onde $\mu$ representa o parâmetro barreira.

$$
\begin{gathered}
L=E F-\sum_{t} \sum_{i} \lambda h_{i}^{t} \cdot\left[V_{i}^{t}+Q_{i}^{t}+S_{i}^{t}-V_{i}^{t-1}-\right. \\
\left.-\sum_{m \in M_{i}}\left(Q_{m}^{t}+S_{m}^{t}\right)-A_{i}^{t}\right]-\sum_{t} \sum_{i} \lambda d^{t} \cdot\left[\sum_{i}\left(P G_{i}^{t}-E F\right)\right]- \\
-\mu \sum_{\mathrm{t}}\left(\sum_{i} \log s l_{i}^{t}-\sum_{i} \log s u_{i}^{t}\right)- \\
-\sum_{t}\left(\sum_{i} \pi l_{i}^{t} \cdot\left(x_{i}^{t}-s l_{i}^{t}-l_{i}\right)-\pi u_{i}^{t} \cdot\left(x_{i}^{t}-s u_{i}^{t}-u_{i}\right)\right)
\end{gathered}
$$

onde,

$\pi l_{i}^{t}$ Representa o multiplicador de Lagrange associado a cada equação de canalização de limite inferior para a usina $i$, durante o estágio $t$;

$\pi u_{i}^{t}$ Representa o multiplicador de Lagrange associado a cada equação de canalização de limite superior para a usina $i$, durante o estágio $t$;

$x_{i}^{t}$ Representa o conjunto de variáveis com limites operativos das usinas $\left(V_{i}^{t}, Q_{i}^{t}\right.$ e $\left.S_{i}^{t}\right)$

A solução do problema linear, resultante da aplicação das condições de Karush-Kuhn-Tucker, associadas ao método de Newton-Raphson é obtida através do método primal-dual de pontos interiores, proposto por (Granville, 1994). Esta referência mostra de forma detalhada um arranjo para a retirada das variáveis de folga (sl e su) e dos multiplicadores de Lagrange $(\pi l$ e $\pi u)$ da solução do problema linear. 
A potência gerada $\left(P G_{i}^{t}\right)$ nas usinas hidrelétricas para cada estágio é determinada através de (3).

$$
P G_{i}^{t}=\rho_{i}^{t} \cdot Q_{i}^{t}
$$

O parâmetro $\rho_{i}^{t}$ representa a produtividade da usina $i$, no estágio $t$ e é dada em MW·mês $/ \mathrm{hm}^{3}$. Quando este parâmetro é considerado constante, o problema (1) é resolvido por programação linear. No entanto, a produtividade é dependente das cotas a jusante e a montante, respectivamente, do reservatório e do canal de fuga. Estes fatores tornam o problema de otimização não linear aumentando a complexidade de solução. A equação (4) mostra como a produtividade varia com as cotas do reservatório.

$$
\rho_{i}^{t}=\rho s p_{i} \cdot\left(\phi_{i}^{t}-\theta_{i}^{t}\right)
$$

onde,

$\rho s p_{i}$ Representa a produtividade específica da usina $i$ [MW. mês $/\left(\mathrm{hm}^{3} \cdot \mathrm{m}\right)$ ];

$\phi_{i}^{t}$ Representa a cota do reservatório na usina $i$, durante o estágio $t[\mathrm{~m}]$;

$\theta_{i}^{t}$ Representa a cota do canal de fuga da usina $i$, durante o estágio $t[\mathrm{~m}]$;

As cotas são representadas através das expressões (5) e (6), e são representados por polinômios de $4^{a}$ ordem.

$$
\begin{gathered}
\phi_{i}^{t}=\sum_{k=1}^{5}\left(C R_{k, i} \cdot\left(V_{i}^{t}\right)^{k-1}\right) \\
\theta_{i}^{t}=\sum_{k=1}^{5}\left(C F_{k, i} \cdot\left(S_{i}^{t}+Q_{i}^{t}\right)^{k-1}\right)
\end{gathered}
$$

onde,

$C R_{k, i}$ Representa o $k^{\text {esimo }}$ coeficiente do polinômio cotavolume da usina $i$;

$C F_{k, i}$ Representa o $k^{e s i m o}$ coeficiente do polinômio VazãoNível Jusante da usina $i$.

A produtividade também depende das perdas hidráulicas nas tubulações da instalação, no entanto neste trabalho elas não serão consideradas.
A inicaliazação das variáveis primais de todas as usinas hidrelétricas foi feita como a seguir: (i) a vazão turbinada sendo igual à metade da vazão máxima turbinável; (ii) vazão vertida igual a zero; (iii) volume armazenado inicial nos reservatórios igual a capacidade máxima de armazenamento dos mesmos; (iv) as variáveis duais foram todas inicializadas iguais a 1 e (v) Energia Firme igual a zero.

A solução do problema (1) fornece, entre outros resultados, o valor da capacidade total do sistema, energia firme global, bem como o período crítico do sistema. A partir destes resultados, pode-se determinar, em uma etapa adicional, o valor da energia firme individualizada das usinas. Para tanto, considera-se a geração média de cada usina hidrelétrica durante o período crítico como a sua energia firme individualizada $\left(E F_{i}\right)$. A energia firme individualizada é, portanto, calculada através de (7).

$$
E F_{i}=\frac{\sum_{t \in C P} P G_{i}^{t}}{N_{C P}}
$$

onde,

$C P$ Representa o conjunto de estágios que englobam o período crítico;

$N_{C P}$ Número de estágios do período crítico.

\section{ASPECTOS COMPUTACIONAIS}

A solução do problema será discutida através de um caso tutorial a fim de possibilitar a análise dos aspectos computacionais do método de pontos interiores. O sistema em análise é composto por 2 usinas e um horizonte de planejamento de 2 meses. A Fig. 1 mostra a configuração em cascata deste sistema.

Para este sistema, a estrutura da matriz Hessiana é definida conforme mostra a Fig. 2. Esta matriz é composta fundamentalmente por duas sub-matrizes, que compõem uma estrutura em blocos de diversos elementos inerentes ao problema com relação às restrições hidráulicas e de demanda.

Na primeira sub-matriz, definida como sub-matriz energética, o bloco de uma usina é composto de quatro elementos, e é representado pelo bloco do tipo $W_{P i}^{S T t}$. Os elementos que compõem este bloco são as variáveis de volume vertido $(S)$, volume turbinado $(Q)$, volume armazenado $(V)$ e o multiplicador de Lagrange correspondente à equação de conservação de água da usina no estágio de planejamento $\left(\lambda_{h}\right)$ e segue esta mesma ordenação na formação do bloco. A seguir é mostrada a construção deste bloco. 


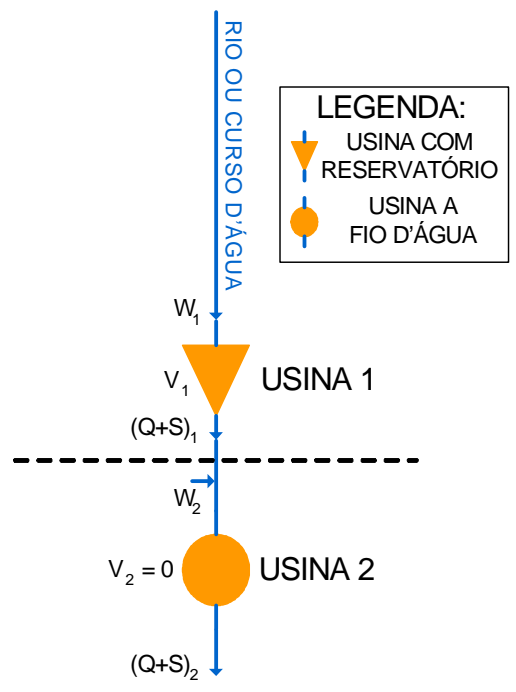

Figura 1: Cascata empregada para estudo tutorial.

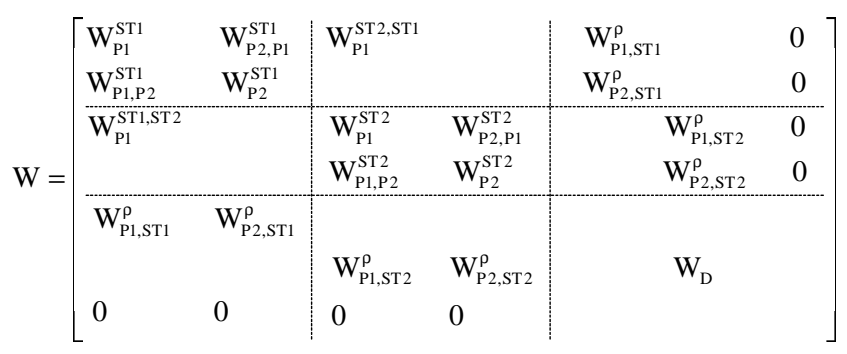

Figura 2: Estrutura da Matriz Hessiana..

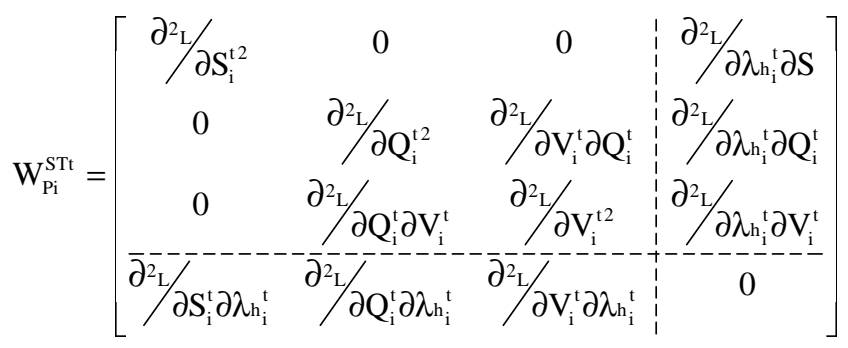

Figura 3: Estrutura do bloco $\mathrm{W}_{P i}^{S T t}$ da Matriz Hessiana.

onde, $L$ denota a equação lagrangeana resultante do problema de otimização, $t$ denota o estágio, e $i$ denota a usina.

Pode-se observar ainda o acoplamento temporal, que representa a interligação do armazenamento de água em uma usina em estágios imediatamente vizinhos, através dos blocos do tipo $W_{P i}^{S T t, S T t+1}$. A seguir é mostrada a construção deste bloco.

O acoplamento hidráulico é observado nos blocos do tipo $W_{P i, P m}^{S T t}$, que representam a interconexão hidráulica entre usinas em uma mesma cascata hídrica, e que associam os vo-
$\mathrm{W}_{\mathrm{Pi}}^{\mathrm{STt}, \mathrm{STt}+1}=\left[\begin{array}{ccc:c}0 & 0 & 0 & 0 \\ 0 & 0 & 0 & 0 \\ 0 & 0 & 0 & 0 \\ \hdashline 0 & 0 & \partial^{2} \mathrm{~L} / \partial \mathrm{V}_{\mathrm{i}}^{\mathrm{t}} \partial \lambda_{\mathrm{i}}^{\mathrm{t}+1} & 0\end{array}\right]$

Figura 4: Estrutura do bloco $\mathrm{W}_{P i}^{S T t, S T t+1}$ da Matriz Hessiana.

lumes vertido e turbinado das usinas imediatamente a montante nas equações de balanço hídrico. A seguir é mostrada a construção deste bloco.

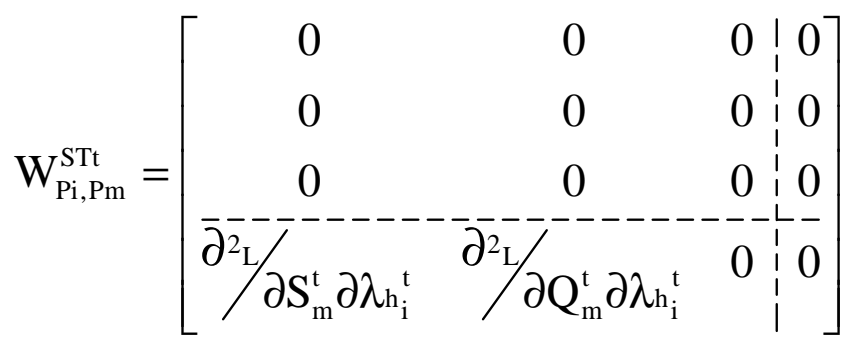

Figura 5: Estrutura do bloco $\mathrm{W}_{P i, P m}^{S T t}$ da Matriz Hessiana.

onde, $m$ denota a usina imediatamente a montate da usina $i$.

A segunda sub-matriz, definida como sub-matriz de demanda, é composta pelos blocos do tipo $W_{P i, S T t}^{\rho}$, e representam a função de produção de energia hidrelétrica, dependente das variáveis de volume vertido $(S)$, volume turbinado $(Q)$, volume armazenado $(V)$ e do multiplicador de Lagrange da equação de conservação de energia correspondente ao estágio $\left(\lambda_{d}\right)$. A seguir é mostrada a construção deste bloco.

$\mathrm{W}_{\mathrm{Pi}, \mathrm{STt}}^{\mathrm{\rho}}=\left[\begin{array}{llll}\partial^{2} \mathrm{~L} / \partial \mathrm{S}_{\mathrm{i}}^{\mathrm{t}} \partial \lambda_{\mathrm{d}_{\mathrm{i}}^{\mathrm{t}}} & \partial^{2} \mathrm{~L} / \partial \mathrm{Q}_{\mathrm{i}}^{\mathrm{t}} \partial \lambda_{\mathrm{d}_{\mathrm{i}}^{\mathrm{t}}} & \partial^{2} \mathrm{~L} / \partial \mathrm{V}_{\mathrm{i}}^{\mathrm{t}} \partial \lambda_{\mathrm{d}_{\mathrm{i}}^{\mathrm{t}}} & 0\end{array}\right]$

Figura 6: Estrutura do bloco $\mathrm{W}_{P i, S T t}^{\rho}$ da Matriz Hessiana.

Finalmente, o bloco de energia firme $\mathrm{W}_{E F}$, é composto pelos multiplicadores de Lagrange da equação de conservação de energia de cada estágio $\left(\lambda_{d}\right)$, ordenados em ordem crescente de estágios, seguido pelo elemento de demanda ou energia firme (EF). A construção deste bloco é mostrada na Figura 7.

As figuras (2-7) enfatizam a esparsidade da matriz Hessiana.

O número de variáveis $(N V)$ do problema pode ser calculado através de (8) de acordo com o número de usinas hidrelétricas e estágios com discretização mensal. 
$\mathrm{W}_{\mathrm{EF}}=\left[\begin{array}{cc:c}0 & 0 & \partial^{2} \mathrm{~L} / \partial \mathrm{EF} \partial \lambda_{\mathrm{d}}^{\mathrm{t}} \\ 0 & 0 & \partial^{2} \mathrm{~L} / \partial \mathrm{EF} \partial \lambda_{\mathrm{d}}^{\mathrm{t}+1} \\ \hdashline \partial^{2} \mathrm{~L} / \partial \lambda_{\mathrm{d}}^{\mathrm{t}} \partial \mathrm{EF} & \partial^{2} \mathrm{~L} / \partial \lambda_{\mathrm{d}}^{\mathrm{t}+1} \partial \mathrm{EF} & \partial^{2} \mathrm{~L} / \partial \mathrm{EF}{ }^{2}\end{array}\right]$

Figura 7: Estrutura do bloco $\mathrm{W}_{E F}$ da Matriz Hessiana.

$$
N V=4 \cdot(N H \cdot N E)+N E+1
$$

onde,

$N E$ Representa o número de estágios de planejamento, com discretização mensal.

Neste caso, utilizando-se a equação (8), tem-se que o número de variáveis de otimização é 19, portanto, a matriz possui 361 elementos. Entretanto, somente 63 são elementos não nulos, ou seja, aproximadamente $17.45 \%$. Para sistemas de grande porte estes elementos são uma parcela menor que $0.01 \%$ do número total de elementos na matriz Hessiana. Esta característica do problema permite a solução do problema de grande porte em um único problema de otimização não linear, utilizando técnicas de esparsidade.

As simulações foram realizadas em um PC Intel Dual Core, $1.86 \mathrm{GHz} / 2048 \mathrm{MB}$ e plataforma C++ em ambiente DOS. As rotinas de ordenação e fatoração utilizadas na implementação computacional foram obtidas através do domínio www.gnu.org.

\section{ESTUDO DE CASO}

Esta seção apresenta uma análise comparativa dos resultados obtidos através da abordagem linear seqüencial (Soares \& Marcato, 2006) e a metodologia proposta.

A simulação foi realizada considerando-se o histórico hidrológico mensal de 70 anos a partir do mês de janeiro de 1931 . A topologia do sistema considera todas as usinas hidrelétricas do Sistema Interligado Nacional (SIN) brasileiro. O SIN em sua configuração do Programa Mensal de Operação (PMO) de janeiro de 2006 é composto por 107 usinas hidrelétricas, contando com 51 usinas com reservatório de regulação e 56 usinas operando a fio d'água. A capacidade total instalada é de 79,50 GW. Os dados cadastrais das usinas hidrelétricas e reservatórios do sistema em estudo e todas as
Tabela 1: Resultados SIN.

\begin{tabular}{|c|c|c|}
\hline Item & $\begin{array}{c}\text { Linear } \\
\text { Seqüencial }\end{array}$ & $\begin{array}{c}\text { Metodologia } \\
\text { Proposta }\end{array}$ \\
\hline $\begin{array}{c}\text { Energia Firme } \\
\text { Global do SIN } \\
\text { (MW) }\end{array}$ & 42570.25 & 42918.15 \\
\hline Período Crítico & $\begin{array}{c}\text { Maio/1952 a } \\
\text { Novembro/1956 }\end{array}$ & $\begin{array}{c}\text { Maio/1952 a } \\
\text { Novembro/1956 }\end{array}$ \\
\hline $\begin{array}{c}\text { Tempo de } \\
\text { Simulação }\end{array}$ & $26 h 40 \mathrm{~min}$ & $1 \mathrm{~h} 46 \mathrm{~min}$ \\
\hline
\end{tabular}

suas características operativas e construtivas foram obtidos através de (CCEE, 2006).

Para o Sistema Interligado Nacional (SIN), a matriz Hessiana associada ao problema de otimização não linear proposto possui uma porcentagem de elementos não nulos de $0,001 \%$. Através de (8) verifica-se que o número de variáveis deste problema é igual a 360.361 unidades. Foram necessárias 259 iterações no método primal-dual de pontos interiores para a convergência.

A energia firme global do SIN e o período crítico são obtidos através de uma única simulação da plataforma computacional proposta. A partir daí são calculados a energia firme individualizada das usinas hidrelétricas e a evolução da energia armazenada no sistema. A Tabela 1 mostra os resultados das simulações realizadas com o modelo linear seqüencial e com o modelo proposto.

Conforme esperado, foi obtida uma diferença na energia firme global de 347,9 MW entre as duas metodologias, o que representa $2,7 \%$. Esta diferença percentual é observada tanto nas usinas a fio d'água quanto nas usinas com reservatório. Em relação ao período crítico, as duas metodologias apontaram o mesmo resultado. Em relação ao tempo computacional, a metodologia proposta apresentou um desempenho muito melhor porque resolve o problema em uma única simulação.

Em relação à energia firme global do sistema, a Figura 8 mostra que as duas metodologias são bastante aderentes. No entanto, observa-se que no início do período crítico o armazenamento do sistema é superior para a metodologia proposta. Este fato justifica o melhor desempenho das usinas do sistema, pois maiores níveis de água nos reservatórios levam o sistema a maiores alturas de queda, o que aumenta a produtividade e a eficiência das usinas hidrelétricas.

Os resultados da metodologia proposta são mais realistas, pois consideram a dependência da produtividade das usinas 


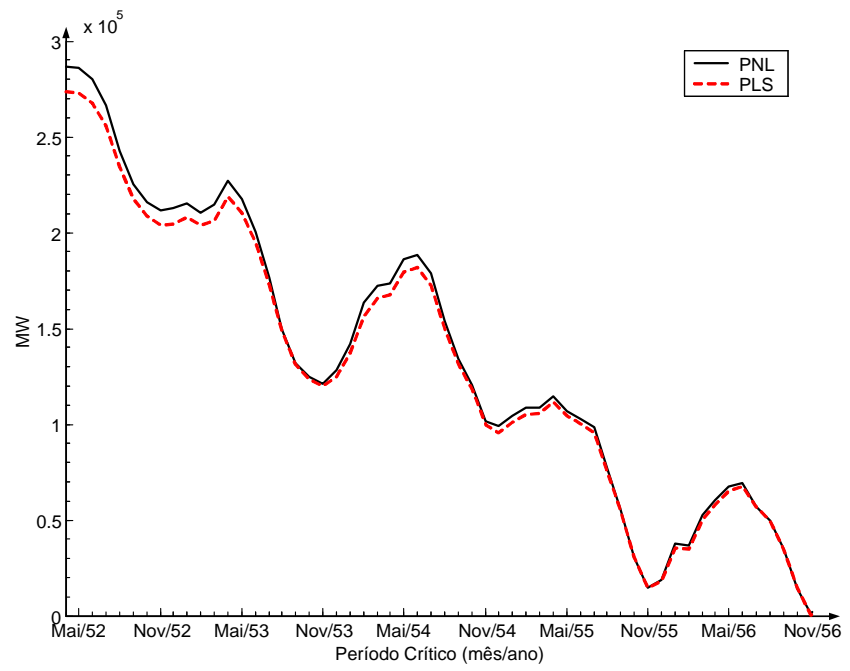

Figura 8: Evolução da Energia armazenada no sistema durante o período crítico.
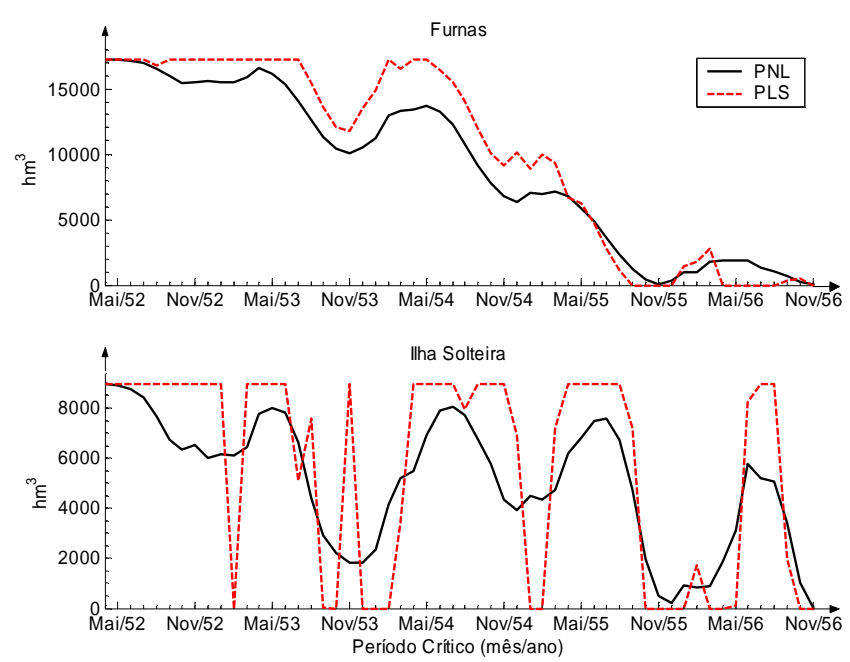

Figura 9: Evolução do Volume Armazenado das Usinas de Furnas e Ilha Solteira Durante o Período Crítico.

em relação aos níveis de montante e jusante. Esta relação entre produtividade e cotas da usina não permite variações bruscas nos níveis dos reservatórios, pois estas variações afetam negativamente a produtividade das usinas. Este fato, contemplado pela metodologia proposta, está em consonância com a prática atual de operação dos reservatórios. A Fig. 9 mostra os resultados obtidos para as usinas de Furnas e Ilha Solteira. Pode-se observar claramente a vantagem da metodologia proposta (PNL).

A Fig. 10 mostra a geração hidráulica durante o período crítico para a Usina de Ilha Solteira. Observa-se que na metodologia proposta ocorreu menos variações da geração tendo em vista o maior equilíbrio de armazenamento. Em conseqüên-

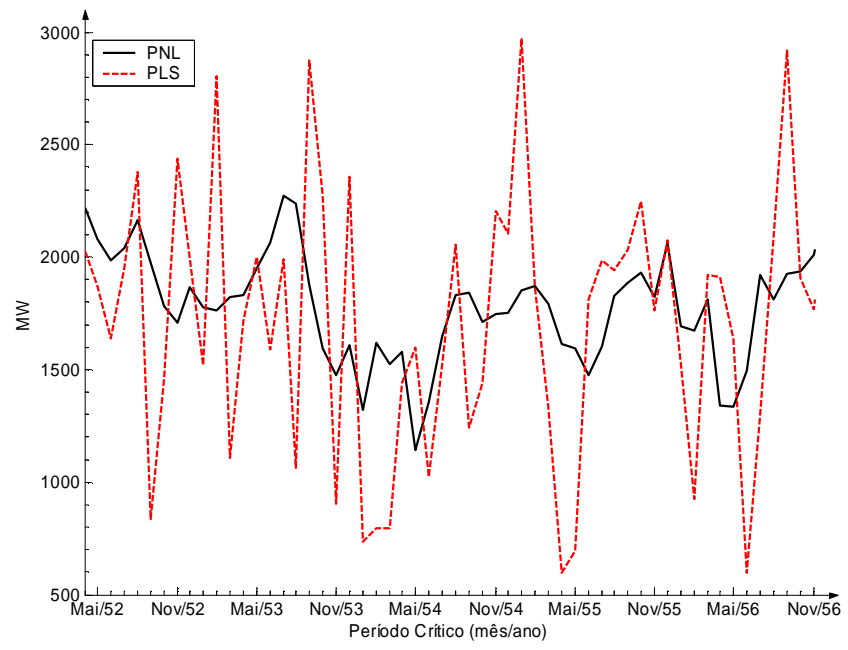

Figura 10: Geração de Ilha Solteira durante o período crítico.
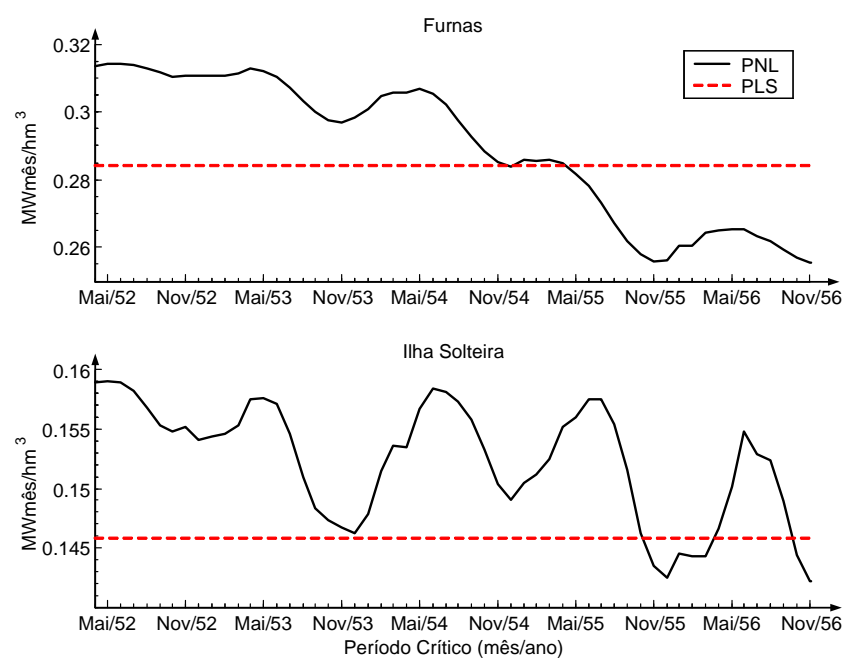

Figura 11: Produtividade das usinas de Furnas e Ilha Solteira durante o período crítico.

cia, a usina apresenta uma geração média maior.

A Fig. 11 apresenta a evolução da produtividade para as usinas Furnas e Ilha Solteira durante o período crítico. Enquanto o modelo linear sequiencial apresenta a produtividade constante, a produtividade real das usinas identificada pela metodologia proposta permanece grande parte do tempo maior.

A Tabela 2 mostra os valores da energia firme individualizado para algumas usinas do SIN e o seu percentual em relação a energia firme global mostrada na Tabela 1. A diferença no percentual de contribuição da Energia Firme individualizada tem impacto direto sobre a garantia física atribuída a cada uma das usinas hidrelétricas. 
Tabela 2: Energia Firme para algumas usinas.

\begin{tabular}{|c|c|c|c|c|}
\hline & \multicolumn{2}{|c|}{$\begin{array}{c}\text { Linear } \\
\text { Seqüencial }\end{array}$} & \multicolumn{2}{c|}{$\begin{array}{c}\text { Metodologia } \\
\text { Proposta }\end{array}$} \\
\hline Usina & $\begin{array}{c}\mathrm{EF} \\
(\mathrm{MW})\end{array}$ & $\begin{array}{c}\mathrm{EF} \\
(\%)\end{array}$ & $\begin{array}{c}\mathrm{EF} \\
(\mathrm{MW})\end{array}$ & $\begin{array}{c}\mathrm{EF} \\
(\%)\end{array}$ \\
\hline Camargos & 18,34 & 0,04 & 15,3 & 0,04 \\
\hline Furnas & 524,33 & 1,23 & 564,47 & 1,32 \\
\hline Marimbondo & 580,33 & 1,36 & 635,29 & 1,48 \\
\hline Ilha Solteira & 1708,8 & 4,01 & 1873,5 & 4,37 \\
\hline Itaipú & 7582,6 & 17,81 & 7718,9 & 18,01 \\
\hline Moxotó & 2382,4 & 5,60 & 2509,1 & 5,85 \\
\hline Tucuruí & 3883,2 & 9,12 & 3828,6 & 8,93 \\
\hline Xingó & 2524,8 & 5,93 & 2686,2 & 6,27 \\
\hline
\end{tabular}

\section{CONCLUSÕES}

Este artigo apresentou uma aplicação do método primal-dual de pontos interiores para o cálculo da energia firme do Sistema Interligado Nacional Brasileiro. A metodologia utilizada permitiu a representação individualizada das usinas hidrelétricas considerando as não linearidades das funções de produtividade. Dos resultados obtidos, os seguintes aspectos podem ser destacados:

- As estimativas das metas de geração são dimensionadas adequadamente ao empregar uma metodologia com maiores detalhamentos do sistema.

- O armazenamento de água nas usinas hidrelétricas tornou-se mais eficiente com a metodologia proposta devido a representação da variação da produtividade das usinas.

- O valor da energia firme encontrado pela metodologia proposta é maior que o valor encontrado pela modelagem linear seqüencial tendo em vista a modelagem mais realista do sistema.

- A energia firme das usinas hidrelétricas sinaliza de forma mais correta a distribuição da energia assegurada conduzindo a um rateio mais justo.

A complexidade da metodologia proposta exigiu a utilização de técnicas de esparsidade para a solução do sistema de equações. O tempo computacional poderá ser reduzido através da utilização de técnicas orientadas a objetos e rotinas de fatoração mais específicas. No entanto, o tempo atual não é fator impeditivo para a utilização da técnica proposta.

\section{DESENVOLVIMENTOS FUTUROS}

A metodologia proposta permite incorporar, no cálculo da energia firme, as não linearidades inerentes ao problema. Portanto, a análise da influência das perdas hidráulicas e da evaporação constituem objetos de pesquisas.

\section{REFERÊNCIAS}

Arvanitids, N. V. and Rosing, J. (1970). Composite representation of multireservoir hydroelectric power system, IEEE Trans. Power Apparatus and Systems, Vol. LXXXIX, No. 2, pp. 319-326.

Câmara de Comercialização de Energia Elétrica (Brasil). Comercialização de Energia - Download do Deck de Preços. Disponível em: <http://www.ccee.org. br/StaticFile/Arquivo/biblioteca_ virtual/Precos/NW200601. zip>. Acesso em: 20 fev. de 2008.

Camargo, I. and Mendes, D. P. (2003). Risk responsibility for supply in the Brazilian energy market, IEEE Power Engineering Society General Meeting, Vol. I, No. 1, pp. 520-524.

Cramton, P. and Stoft, S. (2007). Columbia firm energy market, Proc. 40th International Conference on System Sciences, Hawaii.

Faria, E.; Barroso, L. A.; Kelman, R.; Granville, S.; Pereira, M. V. and Iliadis, N. (2006). Allocation of firm-energy rights among hydro agents using cooperative game theory: an aumann-shapley approach, Operation Research Models and Methods in the Energy Sector, Portugal.

Granville, S. (1994). Optimal reactive dispatch through interior point methods, IEEE Trans. Power Systems, Vol. IX, No. 1, pp. 136-146.

Hicks, R. H.; Gagnon, C. R.; Jacoby, S. L. S. and Kowalik, J. S. (1974). Large scale, nonlinear optimization of energy capability for the pacific northwest hydroelectric system, IEEE Trans. Power Apparatus and Systems, Vol. XCIII, No. 5, pp. 1604-1612.

Hreinsson, E. B. and Barroso, L. A. (2004). Defining optimal production capacity in a purely hydroelectric power system, IEEE International Conference on Electric Utility Deregulation Restructuring and Power Technologies, Hong Kong, Vol. 1, pp. 178-183.

Karmarkar, N. (1984). A New polynomial-time algorithm for linear programming, Combinatorica, Vol. 4, No. 4, pp. 373-395. 
Marques, T. C.; Cicogna, M. A. and Soares, S. (2006). Benefits of coordination in the operation of hydroelectric power systems: Brazilian case, IEEE Power Engineering Society General Meeting, Montreal, Que., pp. 8.

Mendes, D. P. and Santana, E. A. (2003). Regulatory and economic issues regarding the sub-markets in the Brazilian electricity industry, Proc. IEEE Power Tech Conf., Bologna, Vol. III, pp. 6.

Mendes, D. P.; Santana, E. A.; Camargo, I. M. T.; Andrade, C. S. and Nunes, H. R. A. (2003). Modelling issues of the Brazilian market design, IEEE Power Engineering Society General Meeting, Vol. I, pp. 447-452.

Pereira, M. V.; Campodonico, N. and Kelman, R. (1998). Long-term hydro scheduling based on stochastic models, Epsom Conference, Zurique.

Silva, D. F.; Ballini, R.; Soares, S. F. and Carneiro, A. A. F. M. (2003). Interpretação lingüística da operação ótima de uma usina hidroelétrica através de redes neurais nebulosas adaptáveis, SBA Controle e Automação, Vol. XIV, No. 3, pp. 330-337.

Soares, M. P. and Marcato, A. L. M. (2006). Otimização linear seqüencial para cálculo de energia firme das usinas hidrelétricas do sistema interligado nacional, XVI Congresso Brasileiro de Automática (CBA), Salvador, pp. 1962-1967.

Soares, S. and Carneiro, A. A. F. M. (1991). Optimal operation of reservoirs for electric generation, IEEE Trans. Power Delivery, Vol. VI, No. 3, pp. 1101-1107.

Yeh, W. W. G. (1985). Reservoir management and operations models: A state-of-the-art review. Water Resources Research, Vol. XXI, No. 12, pp. 1797-1818.

Yu, Z.; Sparrow, F. T. and Bowen, B. H. (1998). A new longterm hydro production scheduling method for maximizing the profit of hydroelectric systems, IEEE Trans. Power Systems, Vol. XIII, No. 1, pp. 66-71. 